\title{
Nachruf zu Warren Lamb
}

\author{
Susanne Bender
}

A uch wenn im stattlichen Alter von 90 Jahren der Tod nicht eine wirkliche Überraschung ist, so habe ich doch mit großer Bestürzung den Tod von Warren Lamb (28.4.1923-21.1.2014) zur Kenntnis genommen. Er war der letzte Vertreter der ersten prägenden Generation der Tanztherapie. Somit stellt sein Tod auch eine Zäsur in der Geschichte der Tanztherapie dar.

Zeit seines Lebens stand Lamb im Schatten von Rudolf von Laban. Einige der Theorien, die Lamb entwickelt hat, werden manchmal Laban zugeschrieben.

Nachdem Warren Lamb 1946 die Marine verlassen hatte, wurde er Student von Rudolf von Laban. Er war von Labans System der Bewegungsnotation und seiner möglichen Anwendung zur Verhaltensanalyse beeindruckt. Drei Jahre lernte er am Art of Movement Studio in Manchester bei Laban die Bewegungsstudien und assistierte anschließend Laban bei der Bewegungsnotation und -analyse. Er unterstütze Laban auch bei der Entwicklung des Laban Lawrence Personal Effort Assessment, während er gleichzeitig eng mit Laban an der Entwicklung einer Beurteilungsmethode zur Karriereplanung arbeitete und gleichzeitig professioneller Tänzer war.

„Industry from 9 to 11.30 I make observations from 7 interviews of candidates for job study technician position. I then went to the Studio [Art of Movement Studio, S. B.] for Rehearsal which was really a run through of several ballets for the benefit of a man from Leeds
University. I have never danced so shabbily. I knew I would but could do nothing about it: perhaps if I'd had a moment to relax I could have composed myself, but everyone pounced on me, hurried me while I undressed and rushed me into my position for the Stravinsky. Just to make the change-over from industry to dance is exhausting and difficult. When industry has meant 2 hours terrific observation concentration and dance requires as intense but a much varied sort of concentration, one or the other must suffer unless I'm superhuman." (aus Lambs Tagebuch, 15.10.1949, zitiert nach McCaw 2006, 54)

1953 gründete Lamb seine eigene Beratungsfirma, Warren Lamb Associates, wo er den Zusammenhang von Bewegungsparametern und Entscheidungsprozessen untersuchte. Es lag ihm daran, die Bewegungsbegriffe so umzuwandeln, dass auch Manager etwas damit anfangen und sie direkt in ihrer Arbeit umsetzen konnten (Moore 2005).

Kurz vor Labans Tod begann Warren Lamb 1957, die Antriebe und Formen zusammen zu untersuchen, weil er darin eine Komplettierung der Persönlichkeit sah (Davies 2006). Lamb hielt sich in der Notation an das Muster der Antriebsnotation und an die Einteilung von Raum, Kraft und Zeit. Genauso wie die Antriebe in kämpferisch (direkt, stark, schnell) und nachgebend (indirekt, leicht, getragen) aufgeteilt sind, unterteilte er die Formveränderungen der Bewegung in die drei Flächen horizontal, vertikal und sagittal und in 


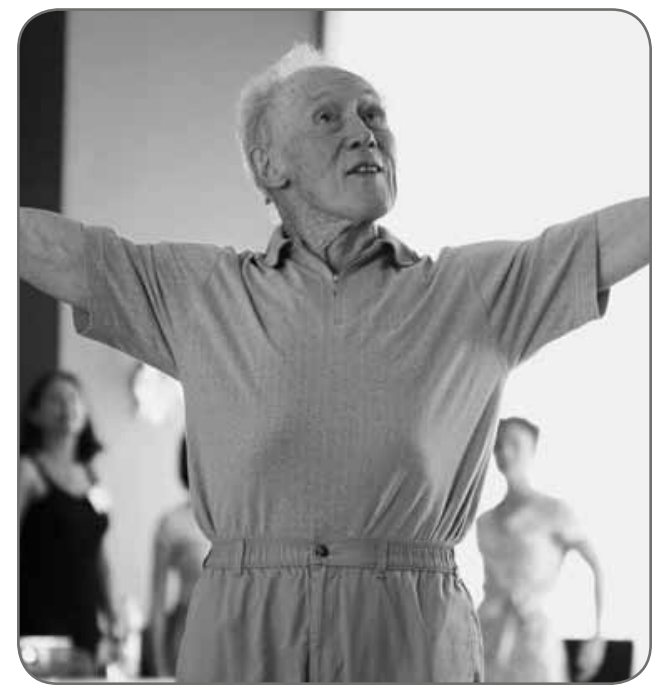

Abb. 1: Warren Lamb beim Kongress Moving from Within im Juli 2010

konkave (nach innen gewölbte) und konvexe (nach außen gewölbte) Gegensatzpaare. Dies übertrug er ebenfalls äquivalent zum Bewegungsfluss auf den Formfluss des Wachsens und Schrumpfens (Lamb 1979). Im englischen Sprachraum (England und USA) wurden diese beiden Ansätze kombiniert zur Effort-ShapeTheorie (Dell 1977).

Nach Labans Tod im Jahr 1958 arbeitete Lamb am Effort-Shape-Konzept weiter und lehrte es einer Vielzahl von Studenten, einschließlich Irmgard Bartenieff und Judith Kestenberg. Seine Hauptarbeit konzentrierte sich jedoch auf die Entwicklung des Action Profiling, was er später, nachdem er einer juristischen Auseinandersetzung unterlegen war, in Movement Pattern Analysis (MPA) umbenannte (Davies 2006). Die Entscheidungsfindung von Managern sollte analysiert werden, um die Ressourcen der Menschen zur Bildung ausgewogener Führungsteams zu optimieren. Viele Führungsteams in über 30 Ländern aus Industrie, Forschung und Bildung wie American Express, Hewlett Packard, IBM, Hambros Bank, Heineken, Kodak and Saatchi \& Saatchi wurden damit erforscht. 2002 analysierte er die Bewegungen von Vladimir Putin und Politikern der US-Regierung.

Neben zahlreichen Artikeln beschreibt er in Posture and Gesture (1965) und Body Code (1979) seine theoretischen Ansätze. Eden Davies (2006) beschreibt in „Beyond Dance: Laban's Legacy of Movement Analysis“ Lambs Lebenswerk.

Zusätzlich zu seinen Beratungstätigkeiten und Lehrtätigkeiten an vielen Universitäten führte er den Vorsitz beim Labanotation Institut an der Universität von Surrey, England.

Warren Lamb war 2007 und 2010 Referent bei Moving from Within, International Congress on Movement Analysis, in Freising bei München, wo ihn die Teilnehmerlnnen als lebendigen, wachen und liebenswerten Lehrer erleben konnten. 2010 wurde er dort für sein Lebenswerk ausgezeichnet.

Aus seiner ersten Ehe hatte er vier Kinder und Enkelkinder. Er war in zweiter Ehe mit seiner Frau Barbara verheiratet und wohnte zuletzt hauptsächlich in Kalifornien, USA.

Er sagte immer, dass er wie Marc Chagall sterben wolle, kreativ arbeitend bis zum letzten Moment. Und so war es auch.

\section{Literatur}

Davies, E. (2006): Beyond dance - Laban's legacy of Movement Analysis. Taylor \& Francis Group, Routledge, New York

Dell, C. (1977): A primer for movement description. Dance Notation Bureau Press, New York

Lamb, W. (1979): Body code. Routledge \& Kegan Paul, London

Lamb, W. (1965): Posture and gesture: An introduction to the study of physical behavior. Brechin Books, London

McCaw, D. (2006): An eye for movement - Warren Lamb's career in Movement Analysis. Brechin Books, London

Moore, C.-L. (2005): Movement and making decisions: The body-mind connection in the workplace. Rosen / Dance \& Movement Press, New York 